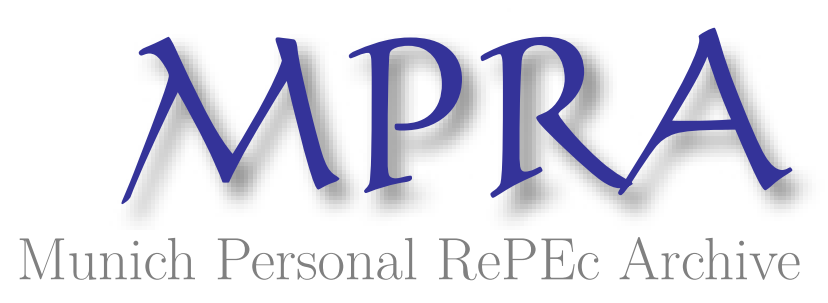

\title{
On foreign aid distortions to governance
}

Asongu, Simplice

4 January 2014

Online at https://mpra.ub.uni-muenchen.de/56812/

MPRA Paper No. 56812, posted 23 Jun 2014 12:56 UTC 


\title{
AFRICAN GOVERNANCE AND DEVELOPMENT INSTITUTE
}

\author{
$\underline{\text { A G D I Working Paper }}$
}

WP/14/003

On foreign aid distortions to governance

Simplice A. Asongu

African Governance and Development Institute, P.O. Box 18 SOA/ 1365 Yaoundé, Cameroon.

E-mail: asongusimplice@yahoo.com 


\title{
AGDI Working Paper
}

Research Department

\section{On foreign aid distortions to governance}

\author{
Simplice A. Asongu ${ }^{1}$
}

January 2014

\begin{abstract}
The Kangoye (2013, TDE) findings on the negative nexus between foreign aid unpredictability and governance could seriously affect debates in academic and policy making circles. Using the theoretical underpinnings of the celebrated Eubank (2012, JDS) literature, we first confirm Kangoye's findings. Then extend the concept of governance from corruption to political, economic, institutional and general versions of the phenomenon. Findings from the extension run counter to those of Kangoye. It follows that in the presence of foreign aid uncertainty, governments could be constrained to improve governance standards in exchange for or anticipation of more dependence on local tax revenues. The empirical evidence is based on 53 African countries for the period 1996-2010. Two direct policy implications result. First, the Kangoye findings for developing countries are relevant for Africa. Second, when the concept of governance is not restricted to corruption, the findings become irrelevant for the continent.

JEL Classification: C53; F35; F47; O11; O55

Keywords: Uncertainty; Foreign aid; Governance; Development; Africa
\end{abstract}

\footnotetext{
${ }^{1}$ Simplice A. Asongu is Lead economist in the Research Department of the AGDI (asongus@afridev.org).
} 


\section{Introduction}

The Kangoye (2013) findings on the negative nexus between foreign aid unpredictability and governance could seriously affect debates in academic and policy making circles. The paper concludes: "This paper examines the effects of aid on governance from a different perspective by asserting that aid unpredictability can potentially increase corruption in recipient countries by providing incentives to risk-averse and corrupt political leaders to engage in rent-seeking activities. Analyses of data from 80 developing countries over the period 1984-2004 offer evidence that higher aid unpredictability is associated with more corruption as measured by a synthetic index. We also find further evidence that this latter impact is more severe in countries with weak initial institutional conditions. These findings are a supplementary advocacy for the need for better management and better predictability of aid flow in developing countries" (p. 121). Our interest in the underlying paper is twofold: restricted use of the concept of governance and a recently celebrated Eubank (2012) hypothesis.

First, restricting the concept of governance to corruption could be misleading. Accordingly, while corruption is employed as the dependent variable of interest, governance is used on the title. We recalibrate the concept of governance into political, economic and institutional dynamics. Consistent with Kaufmann et al. (2010) as recently employed in the literature (Andrés \& Asongu, 2013; Andrés et al., 2014), governance is political (voice \& accountability and political stability/no violence), economic (governance effectiveness and regulation quality), institutional (corruption-control and rule of law) and general (political, economic and institutional). We use these governance dynamics in our study to extend the underpinning paper.

Second, after the Bottom Billion (Collier, 2007) and Dead Aid (Moyo, 2009), inter alia, that have been critically engaged in academic and policy making circles, the Eubank (2012) 
Somaliland-based hypothesis has been recently celebrated with the best paper award from the Journal of Development Studies in 2013. Consistent with the author, the government depends on local taxpayers for revenue in exchange for better governance standards. Hence, according to the narrative, taxpayers have the leverage to demand for better governance standards. The theoretical underpinnings of the hypothesis are deeply rooted in the history of economic thought. It has foundations in negotiations between autocratic governments who needed tax income (to survive inter-state wars) and citizens who were willing only to consent to taxation if there was better governance and delivery of public commodities.

In light of the above, it is interesting to engage how the above two underpinning points converge to give substance to the motivation of this paper. Two main axes of convergence boldly standout: (1) Somaliland is not eligible for foreign aid but has relatively better governance standards and; (2) the Kangoye (2013) findings run counter to the intuition of Eubank (2012).

First, while Somaliland is not eligible to official development assistance, compared to other African nations, it has relatively better governance standards. The increased confidence of Somaliland government in light of the Eubank hypothesis could be summarized in stylized facts from Somaliland's minister of energy and minerals. At a recent African mining conference Hussein Abdi Dualeh professed that his country did not need foreign aid because it was better-off without it: "That is a blessing in disguise. Aid never developed anything...Aid is not a panacea, we'd rather not have it.... How many African countries do you know that developed because of a lot of aid? It's a curse. The ones that get the most aid are the ones with the problems....We've been left to our own devices. We are our own people and our own guys. We pull ourselves up by our own bootstraps. We owe absolutely nothing to anybody. We would not change hands with Greece today. We have zero debt" (Stoddard, 2014). 
Second, the Kangoye findings for developing countries run counter to the Eubank hypothesis which has recently been confirmed in Africa (Asongu, 2014a). Accordingly, foreign aid uncertainty could be assimilated to a situation where domestic government starts anticipating of resorting to more local tax revenues to compensate for the potential negative gap in foreign aid. Hence, the State should be predisposed to improving its governance standards in exchange for or anticipation of more local tax income. The Eubank hypothesis come-in at this juncture. Unfortunately, the Kangoye findings have suggested the contrary. We address this puzzle by postulation that the contradiction is based on the limited scope of the concept of governance, which is restricted to corruption. Hence, we hypothesize that the Eubank hypothesis might be confirmed in more exhaustive contexts of political, economic, institutional and general governance.

The procedure of verifying our postulations consists of three main steps. First, we confirm the Kangoye findings with the concept of corruption as the main indicator of governance. Second, we extend the conception of governance to political, economic, institutional and general dimensions using the six principal government quality dynamics, notably: political stability and voice \& accountability (political); government effectiveness and regulation quality (economic); corruption-control and rule of law (institutional); and general (political, economic \& institutional). Third, we replicate the approach that has confirmed the Kangoye findings to assess if his results are confirmed in the broader spectrum of governance. In other words, if the Eubank intuition is validated.

The rest of the study is organized as follows. Section 2 discusses the data and the methodology. The empirical analysis and discussion of results are covered in Section 3. Section 4 concludes. 


\section{Data and Methodology}

\subsection{Data}

We examine a sample of 53 African countries using annual from African Development Indicators of the World Bank for the period 1996-2010. While the data of Kangoye ranges from 1984 to 2004, good governance indicators from the World Bank only date from 1996. The focus on the African continent has a twofold justification. It is: (1) consistent with developing countries to which the findings of Kangoye are relevant and; (2) in line with the Eubank (2012) hypothesis which has been postulated and confirmed in Africa (Asongu, 2014a).

The dependent variables are measured in terms of the corruption perception index (CPI) and governance dynamics (political, economic, institutional and general). While the CPI is used in baseline analysis to confirm the Kangoye findings, governance dynamics are employed to verify if the findings are valid with an exhaustive conception of governance in light of the Eubank hypothesis. The governance variable are obtained from principal component analysis (PCA) discussed in Section 3.2 below.

The independent variable of interest is net official development assistance (NODA). In order to add subtlety to the analysis for robustness purposes, we add: (1) NODA from the Development Assistance Committee (NODADAC) and; (2) NODA from Multilateral Donors (NODAMD). The distortions are computed using two approaches: (1) simple standard deviations of three-year intervals and; (2) standard errors or standard deviations of the saved residuals after first-order autoregressive processes in the NODA dynamics. The latter approach is consistent with Kangoye.

The choice of three non-overlapping intervals (NOI) has a fourfold justification. First, one degree of freedom is lost after computation of residuals in the first-order autoregressive process and at least two periods are needed for standard deviations of the residuals to be further 
computed. Second, averages mitigate short-run or business cycle disturbances that may loom substantially large. Third, 3 year NOI ensure that the basic conditions for the employment of Generalized Methods of Moments (GMM) are well satisfied (N>T: 53>5). Fourth, 3 year NOI restrict overidentification or limit instrument proliferation by ensuring that the number of crosssections are higher than the number of instruments.

We control for inflation, trade openness, economic prosperity and government expenditure. Whereas the role of government expenditure is consistent with fiscal behavior in governance (Eubank, 2012; Asongu \& Jellal, 2013), globalization in terms of trade openness has been documented to improve governance (Khandelwal \& Roitman, 2012; Asongu, 2014b). Economic prosperity and income-levels are instrumental in the quality of government (Asongu, 2012, p. 191). The sign of inflation on governance remains ambiguous. It may be positive if measures put in place are designed to effectively improve government quality and correct the problem. On the other hand, it could substantially affect governance standards negatively if issues of soaring food prices remain unaddressed. The latter are among factors that culminated to the Arab Spring (Khandelwal \& Roitman, 2012). We also employ time-effects in the specifications to further control for the unobserved heterogeneity.

Definition of the variables is presented in Appendix 1, the summary statistics disclosed in Appendix 2 and the correlation analysis in Appendix 3. From the first, the variables are comparable and based on the variations we could be confident that reasonable estimated relationships would emerge. The correlation analysis has been employed to mitigate multicollinearity and overparametization issues that could arise, especially among NODA distortion dynamics. 


\subsection{Methodology}

The system GMM estimation strategy is adopted for a threefold interest: first, it accounts for potential endogeneity in all the regressors; second, cross-country regressions are eliminated in the estimation process and; biases in the difference estimation resulting from small samples are mitigated. Hence, it is substantially for this third point that we are consistent with Bond et al. (2001, pp. 3-4) in choosing the system GMM approach (Arellano \& Bover, 1995; Blundell \& Bond, 1998) instead of the difference estimator (Arellano \& Bond, 1991). In the specification, a heteroscedasticity-consistent two-step approach is preferred to the homoscedasticity-consistent one-step procedure. Two tests are performed to ascertain the validity of the models, notably: the Sargan over-identifying restrictions (OIR) test for instrument validity and; the Arellano \& Bond autocorrelation $(\mathrm{AR}(2))$ test for the absence of autocorrelation in the residuals. The interests of using data averages in terms of 3 year NOI have already been discussed in the data section.

The following equations in levels and first difference represent the GMM approach.

$$
\begin{aligned}
& \operatorname{Gov}_{i, t}=\sigma_{0}+\sigma_{1} \operatorname{Gov}_{i, t-1}+\sigma_{2} T_{i, t}+\sigma_{3} D A C_{i, t}+\sigma_{4} M D_{i, t}+\sum_{j=1}^{4} \partial_{j} X_{i, t}+\eta_{i}+\xi_{t}+\varepsilon_{i, t} \\
& \operatorname{Gov}_{i, t}-\operatorname{Gov}_{i, t-1}=\sigma_{1}\left(\operatorname{Gov}_{i, t-1}-\operatorname{Gov}_{i, t-2}\right)+\sigma_{2}\left(T_{i, t}-T_{i, t-1}\right)+\sigma_{3}\left(D A C_{i, t}-D A C_{i, t-1}\right) \\
& +\sigma_{4}\left(M D_{i, t}-M D_{i, t-1}\right)+\sum_{j=1}^{4} \partial_{j}\left(X_{i, t}-X_{i, t-1}\right)+\left(\xi_{t}-\xi_{t-1}\right)+\left(\varepsilon_{i, t}-\varepsilon_{i, t-1}\right)
\end{aligned}
$$

Where: 't' denotes the period and ' $\mathrm{i}$ ' stands for a country. Gov is Governance; $T$, Total NODA; $D A C$, NODA from DAC countries; $M D$, NODA from Multilateral Donors; $X$ is the set of control variables (Trade openness, Government expenditure, Inflation and GDP growth); $\eta_{i}$ is a country-specific effect; $\xi_{t}$ is a time-specific constant and; $\varepsilon_{i, t}$ an error term. The estimation procedure involves jointly estimating the regression in levels (Eq. (1)) with that in 
first-difference (Eq. (2)), hence exploiting all the parallel or orthogonality conditions between the the error term and the lagged endogenous variable.

\section{Empirical results}

\subsection{Confirmation of empirical underpinnings}

As outlined in the introduction, it is relevant to first confirm the findings of Kangoye using the same: definition of uncertainty and; corruption as the dependent governance variable. The foreign aid distortions are standard errors (standard deviations of the residuals after firstorder autoregressive processes). In Table 1 below, the models are overwhelmingly valid because the null hypotheses of the Sargan OIR and AR(2) tests are overwhelmingly rejected ${ }^{2}$. The main findings are broadly consistent with the position that foreign aid uncertainty increases corruption. In the interpretation, note should be taken of the fact that the CPI is reported in decreasing order such that, lower values denote higher levels of corruption.

\footnotetext{
${ }^{2}$ Accordingly, two tests have been performed to investigate the validity of the models, notably: the Arellano \& Bond autocorrelation test which investigates the null hypothesis of no autocorrelation and the Sargan-test that assesses the over-identification restrictions. The latter test investigates if the instruments are not correlated with the error term in the equation of interest. The null hypothesis of this test is the view that the instruments as a group display strict exogeneity or do not suffer from endogeneity. Overwhelmingly for most models, we have neither rejected the AR(2) null hypothesis for the absence of autocorrelation nor the Sargan null for the validity of the instruments.
} 
Table 1: Effect of foreign aid distortions on corruption

\begin{tabular}{|c|c|c|c|c|c|c|}
\hline \multirow[b]{2}{*}{ Corruption (-1) } & \multicolumn{6}{|c|}{ Corruption } \\
\hline & $\begin{array}{c}\mathbf{0 . 6 7 0} * * * \\
(\mathbf{0 . 0 0 0 )}\end{array}$ & $\begin{array}{c}\mathbf{0 . 7 0 1} * * * \\
(\mathbf{0 . 0 0 0 )}\end{array}$ & $\begin{array}{c}\mathbf{0 . 6 8 6} * * * \\
(\mathbf{0 . 0 0 0 )}\end{array}$ & $\begin{array}{c}\mathbf{0 . 7 1 4} * * * \\
(\mathbf{0 . 0 0 0 )}\end{array}$ & $\begin{array}{c}\mathbf{0 . 7 1 1} * * * \\
(0.000)\end{array}$ & $\begin{array}{c}\mathbf{0 . 6 7 1} * * * \\
(0.000)\end{array}$ \\
\hline Constant & $\begin{array}{c}0.802 \\
(0.155)\end{array}$ & $\begin{array}{c}0.910 \\
(0.120)\end{array}$ & $\begin{array}{c}0.758 \\
(0.192)\end{array}$ & $\begin{array}{c}0.858 \\
(0.139)\end{array}$ & $\begin{array}{c}0.672 \\
(0.252)\end{array}$ & $\begin{array}{l}0.929 * \\
(0.090)\end{array}$ \\
\hline NODASD2 (Total) & $\begin{array}{l}-0.010 \\
(0.347)\end{array}$ & $\begin{array}{l}-0.003 \\
(0.801)\end{array}$ & --- & --- & -- & --- \\
\hline NODADACSD2 & --- & --- & $\begin{array}{l}-0.010 \\
(0.253)\end{array}$ & $\begin{array}{c}0.000 \\
(0.999)\end{array}$ & --- & --- \\
\hline NODAMDSD2 & --- & --- & --- & --- & $\begin{array}{c}-\mathbf{0 . 0 9 1} * * \\
(\mathbf{0 . 0 3 2})\end{array}$ & $\begin{array}{c}-0.103 * \\
(0.066)\end{array}$ \\
\hline Gov. Expenditure & $\begin{array}{c}-0.003 \\
(0.533)\end{array}$ & $\begin{array}{r}-0.0003 \\
(0.950)\end{array}$ & $\begin{array}{c}-0.002 \\
(0.633)\end{array}$ & $\begin{array}{c}0.000 \\
(0.999)\end{array}$ & $\begin{array}{c}-0.004 \\
(0.150)\end{array}$ & $\begin{array}{l}-0.001 \\
(0.689)\end{array}$ \\
\hline GDP growth & $\begin{array}{l}-0.019 \\
(0.489)\end{array}$ & $\begin{array}{l}-0.005 \\
(0.901)\end{array}$ & $\begin{array}{l}-0.021 \\
(0.437)\end{array}$ & $\begin{array}{c}-0.002 \\
(0.961)\end{array}$ & $\begin{array}{l}-0.008 \\
(0.765)\end{array}$ & $\begin{array}{c}0.003 \\
(0.921)\end{array}$ \\
\hline Trade & $\begin{array}{c}0.004 \\
(0.142)\end{array}$ & $\begin{array}{c}0.004 \\
(0.325)\end{array}$ & $\begin{array}{c}0.004 \\
(0.159)\end{array}$ & $\begin{array}{c}0.004 \\
(0.291)\end{array}$ & $\begin{array}{c}0.004 \\
(0.124)\end{array}$ & $\begin{array}{c}0.004 \\
(0.289)\end{array}$ \\
\hline Inflation & $\begin{array}{c}0.006 \\
(0.784)\end{array}$ & $\begin{array}{c}-0.002 \\
(0.911)\end{array}$ & $\begin{array}{c}0.005 \\
(0.812)\end{array}$ & $\begin{array}{l}-0.005 \\
(0.786)\end{array}$ & $\begin{array}{c}0.010 \\
(0.501)\end{array}$ & $\begin{array}{c}0.007 \\
(0.696)\end{array}$ \\
\hline $\begin{array}{l}\text { Time effects } \\
\operatorname{AR}(2)\end{array}$ & $\begin{array}{c}\text { No } \\
(\mathbf{0 . 2 9 5})\end{array}$ & $\begin{array}{c}\text { Yes } \\
(\mathbf{0 . 4 0 5})\end{array}$ & $\begin{array}{c}\text { No } \\
(\mathbf{0 . 3 1 0})\end{array}$ & $\begin{array}{c}\text { Yes } \\
(\mathbf{0 . 3 7 4})\end{array}$ & $\begin{array}{c}\text { No } \\
(\mathbf{0 . 2 9 9})\end{array}$ & $\begin{array}{c}\text { Yes } \\
(\mathbf{0 . 3 4 3})\end{array}$ \\
\hline Sargan OIR & $(0.511)$ & (0.238) & (0.507) & $(\mathbf{0 . 2 4 7})$ & (0.654) & $(0.212)$ \\
\hline Wald (joint) & $\begin{array}{c}819.47 * * * \\
(0.000)\end{array}$ & $\begin{array}{c}267.53 * * * * \\
(0.000)\end{array}$ & $\begin{array}{c}598.36 * * * * \\
(0.000)\end{array}$ & $\begin{array}{c}273.44 * * * \\
(0.000)\end{array}$ & $\begin{array}{c}\text { 883.72**** } \\
(0.000)\end{array}$ & $\begin{array}{c}259.56 * * * * \\
(0.000)\end{array}$ \\
\hline Instruments & 15 & 18 & 15 & 18 & 15 & 18 \\
\hline Countries & 21 & 21 & 21 & 21 & 21 & 21 \\
\hline Observations & 69 & 69 & 69 & 69 & 69 & 69 \\
\hline
\end{tabular}

$* * *, * *$, and $*$ indicate significance at $1 \%, 5 \%$ and $10 \%$ levels respectively. AR(2): Second Order Autocorrelation test. OIR: Overidentifying Restrictions test. The significance of bold values is twofold. 1) The significance of estimated coefficients and the Wald statistics. 2) The failure to reject the null hypotheses of: a) no autocorrelation in the AR(2) tests and; b) the validity of the instruments in the Sargan OIR test. P-values in bracket. Gov: Government. NODADAC: Net Official Development Assistance from the Development Assistance Committee. NODAMD: Net Official Development Assistance from Multilateral Donors. NODADACSD2: SD2: Distortions by Standard Deviation of the Residuals after first-order autoregressive processes.

\subsection{Requalification of governance}

This section extends the definition of governance from corruption to political, economic, institutional and general dynamics. We use principal component analysis (PCA) to reduce the dimensions of each governance dynamic because some information may be redundant owing to the high degrees of substitution. PCA is a widely employed statistical method that consists of reducing a set of highly correlated variables into a smaller set of uncorrelated variables called principal components that reflect a substantial variation or proportion of initial information. We first reduce all the governance indicators to obtained a general governance measurement before 
further mitigating them into: voce \& accountability and political stability for political governance (PolGov); government effectiveness and regulation quality for economic governance (EcoGov) and; corruption-control and rule of law for institutional governance (InstGov).

The Kaiser (1974) and Jolliffe (2002) criterion are employed to determine common factors. They recommend stopping at first principal components (PCs) with an eigen value greater than the mean (or unity). In this light, as shown in Table 2 below: General governance (G.Gov) has an eigenvalue of 4.642 and represents more than $77 \%$ of variation in the six government variables (regulation quality, government effectiveness, corruption-control, rule of law, political stability/no violence and voice \& accountability); political governance (PolGov) summarizes about $82 \%$ of information with an eigenvalue of 1.852 ; economic governance denotes more than $90 \%$ of information with an eigenvalue of 1.812 and; institutional governance represents $93.5 \%$ of variability with a 1.871 eigenvalue. Consistent with Andrés et al. (2014), the following definitions are relevant to the governance dynamics. (1) Political governance is the process by which those in authority are selected and replaced. (2) Economic governance denotes the capacity of government to formulate \& implement policies as well as deliver services. Institutional governance represents the respect for citizens and the state of institutions that govern the interactions among them.

Table 2: Principal Component Analysis (PCA) for Governance (Gov)

\begin{tabular}{|c|c|c|c|c|c|c|c|c|c|}
\hline $\begin{array}{c}\text { Principal } \\
\text { Components }\end{array}$ & \multicolumn{6}{|c|}{ Component Matrix(Loadings) } & \multirow[t]{2}{*}{ Proportion } & \multirow{2}{*}{$\begin{array}{l}\text { Cumulative } \\
\text { Proportion }\end{array}$} & \multirow{2}{*}{$\begin{array}{l}\text { Eigen } \\
\text { Value } \\
\end{array}$} \\
\hline & VA & PS & $\mathbf{R Q}$ & GE & RL & $\mathbf{C C}$ & & & \\
\hline First PC (G.Gov) & 0.383 & 0.374 & 0.403 & 0.429 & 0.443 & 0.413 & 0.773 & 0.773 & 4.642 \\
\hline Second PC & 0.297 & 0.774 & -0.369 & -0.350 & -0.021 & -0.230 & 0.077 & 0.851 & 0.466 \\
\hline Third PC & 0.750 & -0.300 & 0.353 & -0.127 & -0.223 & -0.396 & 0.066 & 0.917 & 0.398 \\
\hline First PC (PolGov) & 0.707 & 0.707 & --- & --- & --- & --- & 0.829 & 0.829 & 1.659 \\
\hline Second PC & -0.707 & 0.707 & --- & --- & --- & --- & 0.170 & 1.000 & 0.340 \\
\hline First PC (EcoGov) & --- & --- & 0.707 & 0.707 & --- & --- & 0.906 & 0.906 & 1.812 \\
\hline Second PC & --- & --- & -0.707 & 0.707 & --- & --- & 0.093 & 1.000 & 0.187 \\
\hline First PC (InstGov) & --- & --- & --- & --- & 0.707 & 0.707 & 0.935 & 0.935 & 1.871 \\
\hline Second PC & --- & --- & --- & --- & -0.707 & 0.707 & 0.064 & 1.000 & 0.128 \\
\hline
\end{tabular}


P.C: Principal Component. VA: Voice \& Accountability. RL: Rule of Law. R.Q: Regulation Quality. GE: Government Effectiveness. PS: Political Stability. CC: Control of Corruption. G.Gov (General Governance): First PC of VA, PS, RQ, GE, RL \& CC. PolGov (Political Governance): First PC of VA \& PS. EcoGov (Economic Governance): First PC of RQ \& GE. InstGov (Institutional Governance): First PC of RL \& CC.

\subsection{Distortions as standard deviations}

Table 3 below assesses the concerns underpinning the paper using the first definition of distortions which is the standard deviation of three-year NOI. But for a thin exception (first model on general governance with a significant Sargan OIR test), the models are overwhelmingly valid because the null hypotheses of the AR(2) and Sargan OIR tests are not rejected for the most part. The main findings support the Eubank hypothesis with a positive effect of aid distortions on political and general governance. Most of the control variables have the expected though insignificant signs.

Table 3: Total foreign aid distortions with standard deviations

\begin{tabular}{|c|c|c|c|c|c|c|c|c|}
\hline \multirow[b]{3}{*}{ Gov $(-1)$} & \multicolumn{8}{|c|}{ Dependent variable: Governance } \\
\hline & \multicolumn{2}{|c|}{$\begin{array}{c}\text { Political Governance } \\
\text { (PolGov) }\end{array}$} & \multicolumn{2}{|c|}{$\begin{array}{c}\text { Economic Governance } \\
(\text { EcoGov })\end{array}$} & \multicolumn{2}{|c|}{$\begin{array}{c}\text { Institutional } \\
\text { Governance (InstGov) }\end{array}$} & \multicolumn{2}{|c|}{$\begin{array}{c}\text { General Governance } \\
\text { (G.Gov) }\end{array}$} \\
\hline & $\begin{array}{l}\mathbf{0 . 9 7 0 * * *} \\
(\mathbf{0 . 0 0 0 )}\end{array}$ & $\begin{array}{l}1.128 * * * \\
(0.000)\end{array}$ & $\begin{array}{l}1.081 * * * \\
(0.000)\end{array}$ & $\begin{array}{l}\mathbf{0 . 8 6 2 * * * *} \\
(\mathbf{0 . 0 0 0 )}\end{array}$ & $\begin{array}{l}\mathbf{0 . 8 5 4} * * * \\
(\mathbf{0 . 0 0 0 )}\end{array}$ & $\begin{array}{l}0.913 * * * \\
(0.000)\end{array}$ & $\begin{array}{l}1.040 * * * \\
(0.000)\end{array}$ & $\begin{array}{l}0.955 * * * \\
(0.000)\end{array}$ \\
\hline Constant & $\begin{array}{l}-0.067 \\
0.358)\end{array}$ & -0.145 & -0.041 & -0.007 & 0.048 & 0.063 & 0.008 & -0.088 \\
\hline NODASD1 (Total) & $\begin{array}{l}(0.358) \\
\text { 0.006* } \\
(\mathbf{0 . 0 5 4 )}\end{array}$ & $\begin{array}{l}(0.177) \\
\mathbf{0 . 0 1 5} * \\
(\mathbf{0 . 0 7 5 )}\end{array}$ & $\begin{array}{l}(0.701) \\
0.012 \\
(0.251)\end{array}$ & $\begin{array}{l}(0.963) \\
0.007 \\
(0.582)\end{array}$ & $\begin{array}{l}(0.572) \\
0.003 \\
(0.482)\end{array}$ & $\begin{array}{l}(0.673) \\
0.006 \\
(0.350)\end{array}$ & $\begin{array}{l}(0.944) \\
\mathbf{0 . 0 1 7} * * \\
(\mathbf{0 . 0 4 4})\end{array}$ & $\begin{array}{l}(0.634) \\
\mathbf{0 . 0 1 7} * \\
(\mathbf{0 . 0 5 6})\end{array}$ \\
\hline Gov. Expenditure & --- & $\begin{array}{l}0.005 \\
(0.324)\end{array}$ & --- & $\begin{array}{l}0.004 \\
(0.539)\end{array}$ & --- & $\begin{array}{l}0.0008 \\
(0.865)\end{array}$ & --- & $\begin{array}{l}0.009 \\
(0.225)\end{array}$ \\
\hline GDP growth & --- & $\begin{array}{l}0.012 \\
(0.217)\end{array}$ & --- & $\begin{array}{l}0.018 \\
(0.240)\end{array}$ & --- & $\begin{array}{l}0.004 \\
(0.786)\end{array}$ & --- & $\begin{array}{l}0.019 \\
(0.383)\end{array}$ \\
\hline Trade & --- & $\begin{array}{l}0.0002 \\
(0.831)\end{array}$ & --- & $\begin{array}{l}0.0005 \\
(0.668)\end{array}$ & --- & $\begin{array}{l}-0.0003 \\
(0.797)\end{array}$ & --- & $\begin{array}{l}0.0007 \\
(0.634)\end{array}$ \\
\hline Inflation & --- & $\begin{array}{l}0.0009 \\
(0.139)\end{array}$ & --- & $\begin{array}{l}-0.001 \\
(0.571)\end{array}$ & --- & $\begin{array}{l}0.001 \\
(0.233)\end{array}$ & --- & $\begin{array}{l}0.0007 \\
(0.393)\end{array}$ \\
\hline $\begin{array}{l}\text { Time effects } \\
\operatorname{AR}(2)\end{array}$ & $\begin{array}{r}\text { Yes } \\
(\mathbf{0 . 5 5 0})\end{array}$ & $\begin{array}{r}\text { Yes } \\
(\mathbf{0 . 6 1 4})\end{array}$ & $\begin{array}{r}\text { Yes } \\
(\mathbf{0 . 3 9 5})\end{array}$ & $\begin{array}{r}\text { Yes } \\
(\mathbf{0 . 7 0 1 )}\end{array}$ & $\begin{array}{r}\text { Yes } \\
(\mathbf{0 . 3 8 9})\end{array}$ & $\begin{array}{r}\text { Yes } \\
(\mathbf{0 . 3 0 0 )}\end{array}$ & $\begin{array}{l}\text { Yes } \\
(\mathbf{0 . 5 2 4 )}\end{array}$ & $\begin{array}{l}\text { Yes } \\
(\mathbf{0 . 3 3 8 )}\end{array}$ \\
\hline Sargan OIR & $(0.599)$ & $(0.290)$ & $(\mathbf{0 . 0 2 9})$ & $(\mathbf{0 . 1 9 6})$ & $(\mathbf{0 . 1 1 5})$ & $(\mathbf{0 . 2 9 7})$ & $(0.071)$ & $(0.252)$ \\
\hline Wald (joint) & $\begin{array}{l}91.426 * * * \\
(0.000)\end{array}$ & $\begin{array}{l}\text { 953.30**** } \\
(\mathbf{0 . 0 0 0 )}\end{array}$ & $\begin{array}{l}102.44 * * * \\
(0.000)\end{array}$ & $\begin{array}{l}1084.3^{* * *} \\
(0.000)\end{array}$ & $\begin{array}{l}\text { 79.441*** } \\
(\mathbf{0 . 0 0 0 )}\end{array}$ & $\begin{array}{l}1339.6^{* * *} \\
(0.000)\end{array}$ & $\begin{array}{l}168.15 * * * \\
(0.000)\end{array}$ & $\begin{array}{l}\text { 3076.3*** } \\
(\mathbf{0 . 0 0 0 )}\end{array}$ \\
\hline Instruments & 14 & 18 & 14 & 18 & 14 & 18 & 14 & 18 \\
\hline Countries & 51 & 34 & 50 & 34 & 51 & 34 & 50 & 34 \\
\hline Observations & 199 & 118 & 195 & 118 & 199 & 118 & 195 & 118 \\
\hline
\end{tabular}


In Table 4 below, specifications of Table 3 are replicated with NODA from DAC countries (Panel A) and NODA from Multilateral Donors (Panel B). The models in both panels are overwhelmingly valid and the underlying Eubank is also confirmed from political and general governance perspectives.

Table 4: DAC and MD foreign aid distortions with standard deviations

\begin{tabular}{|c|c|c|c|c|c|c|c|c|}
\hline \multirow[b]{3}{*}{ Gov (-1) } & \multicolumn{8}{|c|}{$\begin{array}{c}\text { Dependent variable: Governance } \\
\text { Panel A: Foreign Aid from the Development Assistance Committee (DAC) Countries }\end{array}$} \\
\hline & \multicolumn{2}{|c|}{$\begin{array}{c}\text { Political Governance } \\
\text { (PolGov) }\end{array}$} & \multicolumn{2}{|c|}{$\begin{array}{l}\text { Economic Governance } \\
\text { (EcoGov) }\end{array}$} & \multicolumn{2}{|c|}{$\begin{array}{c}\text { Institutional } \\
\text { Governance (InstGov) }\end{array}$} & \multicolumn{2}{|c|}{$\begin{array}{l}\text { General Governance } \\
\text { (G.Gov) }\end{array}$} \\
\hline & $\begin{array}{l}0.992 * * * \\
(0.000)\end{array}$ & $\begin{array}{l}\text { 1.131*** } \\
(\mathbf{0 . 0 0 0 )}\end{array}$ & $\begin{array}{l}\text { 1.084*** } \\
(\mathbf{0 . 0 0 0 )}\end{array}$ & $\begin{array}{l}\mathbf{0 . 8 3 5} * * * \\
(\mathbf{0 . 0 0 0 )}\end{array}$ & $\begin{array}{l}\mathbf{0 . 8 4 3} * * * \\
(\mathbf{0 . 0 0 0 )}\end{array}$ & $\begin{array}{l}\text { 0.919**** } \\
(\mathbf{0 . 0 0 0 )}\end{array}$ & $\begin{array}{l}1.035 * * * \\
(\mathbf{0 . 0 0 0 )}\end{array}$ & $\begin{array}{l}0.963 * * * \\
(0.000)\end{array}$ \\
\hline Constant & $\begin{array}{l}-0.054 \\
(0.467)\end{array}$ & $\begin{array}{l}-0.160 \\
(0.140)\end{array}$ & $\begin{array}{l}-0.044 \\
(0.677)\end{array}$ & $\begin{array}{l}0.009 \\
(0.952)\end{array}$ & $\begin{array}{l}0.055 \\
(0.526)\end{array}$ & $\begin{array}{l}0.081 \\
(0.581)\end{array}$ & $\begin{array}{l}0.022 \\
(0.844)\end{array}$ & $\begin{array}{l}-0.081 \\
(0.670)\end{array}$ \\
\hline NODADACSD1 & $\begin{array}{l}0.002 \\
(0.541)\end{array}$ & $\begin{array}{l}0.019 * * * \\
(0.000)\end{array}$ & $\begin{array}{l}0.016 \\
(0.249)\end{array}$ & $\begin{array}{l}0.005 \\
(0.714)\end{array}$ & $\begin{array}{l}0.0009 \\
(0.844)\end{array}$ & $\begin{array}{l}0.004 \\
(0.513)\end{array}$ & $\begin{array}{l}\text { 0.019* } \\
(0.092)\end{array}$ & $\begin{array}{l}\text { 0.016** } \\
(0.041)\end{array}$ \\
\hline Gov. Expenditure & --- & $\begin{array}{l}0.005 \\
(0.299)\end{array}$ & --- & $\begin{array}{l}0.004 \\
(0.617)\end{array}$ & --- & $\begin{array}{l}0.0007 \\
(0.873)\end{array}$ & --- & $\begin{array}{l}0.009 \\
(0.216)\end{array}$ \\
\hline GDP growth & --- & $\begin{array}{l}0.013 \\
(0.188)\end{array}$ & --- & $\begin{array}{l}0.019 \\
(0.223)\end{array}$ & --- & $\begin{array}{l}0.004 \\
(0.771)\end{array}$ & --- & $\begin{array}{l}0.019 \\
(0.357)\end{array}$ \\
\hline Trade & --- & $\begin{array}{l}0.0003 \\
(0.752)\end{array}$ & --- & $\begin{array}{l}0.0006 \\
(0.605)\end{array}$ & --- & $\begin{array}{l}-0.0004 \\
(0.740)\end{array}$ & --- & $\begin{array}{l}0.0006 \\
(0.686)\end{array}$ \\
\hline Inflation & --- & $\begin{array}{l}\mathbf{0 . 0 0 1} * \\
(\mathbf{0 . 0 8 4 )}\end{array}$ & --- & $\begin{array}{l}-0.001 \\
(0.530)\end{array}$ & --- & $\begin{array}{l}0.001 \\
(0.272)\end{array}$ & --- & $\begin{array}{l}0.0008 \\
(0.355)\end{array}$ \\
\hline $\begin{array}{l}\text { Time effects } \\
\operatorname{AR}(2)\end{array}$ & $\begin{array}{l}\text { Yes } \\
(\mathbf{0 . 6 4 6 )}\end{array}$ & $\begin{array}{l}\text { Yes } \\
(\mathbf{0 . 6 6 9 )}\end{array}$ & $\begin{array}{l}\text { Yes } \\
(\mathbf{0 . 3 3 8 )}\end{array}$ & $\begin{array}{l}\text { Yes } \\
(\mathbf{0 . 6 8 2})\end{array}$ & $\begin{array}{l}\text { Yes } \\
(\mathbf{0 . 3 8 3 )}\end{array}$ & $\begin{array}{l}\text { Yes } \\
(\mathbf{0 . 2 9 6 )}\end{array}$ & $\begin{array}{l}\text { Yes } \\
(\mathbf{0 . 5 6 0 )}\end{array}$ & $\begin{array}{l}\text { Yes } \\
(\mathbf{0 . 3 3 3 )}\end{array}$ \\
\hline Sargan OIR & $(0.536)$ & $(0.281)$ & $(0.032)$ & $(0.199)$ & $(0.120)$ & $(\mathbf{0 . 3 3 0})$ & $(0.075)$ & $(0.244)$ \\
\hline Wald (joint) & $\begin{array}{l}50.416 * * * \\
(0.000)\end{array}$ & $\begin{array}{l}1245.2 * * * * \\
(0.000)\end{array}$ & $\begin{array}{l}\mathbf{1 1 2 . 7 0}^{* * * *} \\
(\mathbf{0 . 0 0 0 )}\end{array}$ & $\begin{array}{l}1085.5^{* * * *} \\
(0.000)\end{array}$ & $\begin{array}{l}\text { 48.786**** } \\
(0.000)\end{array}$ & $\begin{array}{l}1674.0 * * * * \\
(0.000)\end{array}$ & $\begin{array}{l}150.14 * * * * \\
(0.000)\end{array}$ & $\begin{array}{l}\text { 2994**** } \\
(0.000)\end{array}$ \\
\hline Instruments & 14 & 18 & 14 & 18 & 14 & 18 & 14 & 18 \\
\hline Countries & 51 & 34 & 50 & 34 & 55 & 34 & 50 & 34 \\
\hline Observations & 199 & 118 & 195 & 118 & 199 & 118 & 195 & 118 \\
\hline
\end{tabular}




\begin{tabular}{|c|c|c|c|c|c|c|c|c|}
\hline \multirow[b]{3}{*}{ Gov $(-1)$} & \multicolumn{8}{|c|}{ Panel B: Foreign Aid from Multilateral Donors } \\
\hline & \multicolumn{2}{|c|}{$\begin{array}{c}\text { Political Governance } \\
\text { (PolGov) }\end{array}$} & \multicolumn{2}{|c|}{$\begin{array}{c}\text { Economic Governance } \\
\text { (EcoGov) }\end{array}$} & \multicolumn{2}{|c|}{$\begin{array}{c}\text { Institutional } \\
\text { Governance (InstGov) }\end{array}$} & \multicolumn{2}{|c|}{$\begin{array}{c}\text { General Governance } \\
\text { (G.Gov) }\end{array}$} \\
\hline & $\begin{array}{l}0.872 * * * \\
(\mathbf{0 . 0 0 0 )}\end{array}$ & $\begin{array}{l}1.115 * * * \\
(0.000)\end{array}$ & $\begin{array}{l}1.047 * * * \\
(0.000)\end{array}$ & $\begin{array}{l}\mathbf{0 . 8 8 2} * * * \\
(\mathbf{0 . 0 0 0 )}\end{array}$ & $\begin{array}{l}\mathbf{0 . 8 3 4} * * * \\
(\mathbf{0 . 0 0 0 )}\end{array}$ & $\begin{array}{l}0.971 * * * \\
(0.000)\end{array}$ & $\begin{array}{l}1.005 * * * \\
(0.000)\end{array}$ & $\begin{array}{l}0.947 * * * \\
(0.000)\end{array}$ \\
\hline Constant & $\begin{array}{l}-0.078 \\
(0.284)\end{array}$ & $\begin{array}{l}-0.106 \\
(0.378)\end{array}$ & $\begin{array}{l}-0.041 \\
(0.705)\end{array}$ & $\begin{array}{l}-0.037 \\
(0.807)\end{array}$ & $\begin{array}{l}0.039 \\
(0.644)\end{array}$ & $\begin{array}{l}0.155 \\
(0.309)\end{array}$ & $\begin{array}{l}-0.014 \\
(0.904)\end{array}$ & $\begin{array}{l}-0.020 \\
(0.903)\end{array}$ \\
\hline NODAMDSD1 & $\begin{array}{l}\mathbf{0 . 0 3 9} * * * \\
(\mathbf{0 . 0 0 0 )}\end{array}$ & $\begin{array}{l}0.011 \\
(0.748)\end{array}$ & $\begin{array}{l}0.025 * * \\
(0.044)\end{array}$ & $\begin{array}{l}0.039 \\
(0.422)\end{array}$ & $\begin{array}{l}0.013 \\
(0.211)\end{array}$ & $\begin{array}{l}0.009 \\
(0.724)\end{array}$ & $\begin{array}{l}\mathbf{0 . 0 5 2} * * * \\
(\mathbf{0 . 0 0 0 )}\end{array}$ & $\begin{array}{l}0.032 \\
(0.493)\end{array}$ \\
\hline Gov. Expenditure & --- & $\begin{array}{l}0.004 \\
(0.390)\end{array}$ & --- & $\begin{array}{l}0.003 \\
(0.646)\end{array}$ & --- & $\begin{array}{l}0.001 \\
(0.711)\end{array}$ & --- & $\begin{array}{l}0.007 \\
(0.283)\end{array}$ \\
\hline GDP growth & --- & $\begin{array}{l}0.013 \\
(0.224)\end{array}$ & --- & $\begin{array}{l}0.020 \\
(0.194)\end{array}$ & --- & $\begin{array}{l}-0.002 \\
(0.865)\end{array}$ & --- & $\begin{array}{l}0.017 \\
(0.462)\end{array}$ \\
\hline Trade & --- & $\begin{array}{l}0.0001 \\
(0.881)\end{array}$ & --- & $\begin{array}{l}0.0004 \\
(0.694)\end{array}$ & --- & $\begin{array}{l}-0.001 \\
(0.465)\end{array}$ & --- & $\begin{array}{l}0.0006 \\
(0.723)\end{array}$ \\
\hline Inflation & --- & $\begin{array}{l}0.0001 \\
(0.743)\end{array}$ & --- & $\begin{array}{l}-0.001 \\
(0.300)\end{array}$ & --- & $\begin{array}{l}0.001 \\
(0.178)\end{array}$ & --- & $\begin{array}{l}-0.0002 \\
(0.678)\end{array}$ \\
\hline $\begin{array}{l}\text { Time effects } \\
\operatorname{AR}(2)\end{array}$ & $\begin{array}{l}\text { Yes } \\
(\mathbf{0 . 5 1 9 )}\end{array}$ & $\begin{array}{l}\text { Yes } \\
(\mathbf{0 . 7 0 0 )}\end{array}$ & $\begin{array}{l}\text { Yes } \\
(\mathbf{0 . 6 0 5 )}\end{array}$ & $\begin{array}{l}\text { Yes } \\
(\mathbf{0 . 9 0 2 )}\end{array}$ & $\begin{array}{l}\text { Yes } \\
(\mathbf{0 . 4 0 6 )}\end{array}$ & $\begin{array}{l}\text { Yes } \\
(\mathbf{0 . 3 3 9 )}\end{array}$ & $\begin{array}{l}\text { Yes } \\
(\mathbf{0 . 6 3 3 )}\end{array}$ & $\begin{array}{l}\text { Yes } \\
(\mathbf{0 . 3 6 4 )}\end{array}$ \\
\hline Sargan OIR & $(\mathbf{0 . 5 6 2 )}$ & $(\mathbf{0 . 2 5 0 )}$ & $(0.035)$ & $(\mathbf{0 . 3 1 9})$ & $(\mathbf{0 . 1 1 4})$ & $(\mathbf{0 . 5 6 2 )}$ & $(0.059)$ & $(\mathbf{0 . 3 9 6 )}$ \\
\hline Wald (joint) & $\begin{array}{l}\text { 59.108**** } \\
(\mathbf{0 . 0 0 0 )}\end{array}$ & $\begin{array}{l}\text { 733.31**** } \\
(\mathbf{0 . 0 0 0 )}\end{array}$ & $\begin{array}{l}156.94 * * * \\
(0.000)\end{array}$ & $\begin{array}{l}1280.6 * * * \\
(0.000)\end{array}$ & $\begin{array}{l}\text { 74.766**** } \\
(0.000)\end{array}$ & $\begin{array}{l}1051.6 * * * * \\
(0.000)\end{array}$ & $\begin{array}{l}153.56 * * * \\
(0.000)\end{array}$ & $\begin{array}{l}2885 * * * * \\
(0.000)\end{array}$ \\
\hline Instruments & 14 & 18 & 14 & 18 & 14 & 18 & 14 & 18 \\
\hline Countries & 51 & 34 & 50 & 34 & 51 & 34 & 50 & 34 \\
\hline Observations & 199 & 118 & 195 & 118 & 199 & 118 & 195 & 118 \\
\hline
\end{tabular}

***,**, and * indicate significance at 1\%,5\% and 10\% levels respectively. AR(2): Second Order Autocorrelation test. OIR: Overidentifying Restrictions test. The significance of bold values is twofold. 1) The significance of estimated coefficients and the Wald statistics. 2) The failure to reject the null hypotheses of: a) no autocorrelation in the AR(2) tests and; b) the validity of the instruments in the Sargan OIR test. P-values in bracket. Gov: Government. NODADAC: Net Official Development Assistance from the Development Assistance Committee. NODAMD: Net Official Development Assistance from Multilateral Donors. NODADAC SD1: Distortions by Simple Standard Deviation.

\subsection{Distortions as standard errors}

Tables 5-6 below address the underlying problem using standard errors as distortions instead of standard deviations. The standard errors are computed as the standard deviations of the residuals saved from the first-order autoregressive processes. The Eubank hypothesis is broadly confirmed for political and general governance. 
Table 5: Total foreign aid distortions with standard errors

\begin{tabular}{|c|c|c|c|c|c|c|c|c|}
\hline \multirow[b]{3}{*}{ Gov $(-1)$} & \multicolumn{8}{|c|}{ Dependent variable: Governance } \\
\hline & \multicolumn{2}{|c|}{$\begin{array}{c}\text { Political Governance } \\
\text { (PolGov) }\end{array}$} & \multicolumn{2}{|c|}{$\begin{array}{c}\text { Economic Governance } \\
\text { (EcoGov) }\end{array}$} & \multicolumn{2}{|c|}{$\begin{array}{c}\text { Institutional } \\
\text { Governance (InstGov) } \\
\end{array}$} & \multicolumn{2}{|c|}{$\begin{array}{c}\text { General Governance } \\
\text { (G.Gov) }\end{array}$} \\
\hline & $\begin{array}{l}0.969 * * * \\
(0.000)\end{array}$ & $\begin{array}{l}1.128 * * * \\
(0.000)\end{array}$ & $\begin{array}{l}1.078 * * * \\
(0.000)\end{array}$ & $\begin{array}{l}\mathbf{0 . 8 2 8} * * * \\
(0.000)\end{array}$ & $\begin{array}{l}\mathbf{0 . 8 4 1} * * * \\
(\mathbf{0 . 0 0 0 )}\end{array}$ & $\begin{array}{l}0.915 * * * \\
(0.000)\end{array}$ & $\begin{array}{l}1.034 * * * \\
(0.000)\end{array}$ & $\begin{array}{l}0.938 * * * \\
(0.000)\end{array}$ \\
\hline Constant & $\begin{array}{l}-0.057 \\
(0.431)\end{array}$ & $\begin{array}{l}-0.147 \\
(0.165)\end{array}$ & $\begin{array}{l}-0.025 \\
(0.815)\end{array}$ & $\begin{array}{l}0.012 \\
(0.938)\end{array}$ & $\begin{array}{l}0.055 \\
(0.513)\end{array}$ & $\begin{array}{l}0.078 \\
(0.593)\end{array}$ & $\begin{array}{l}0.030 \\
(0.785)\end{array}$ & $\begin{array}{l}-0.068 \\
(0.691)\end{array}$ \\
\hline NODA SD2 (Total) & $\begin{array}{l}\text { 0.003** } \\
(\mathbf{0 . 0 3 0 )}\end{array}$ & $\begin{array}{l}\text { 0.012** } \\
(\mathbf{0 . 0 3 5 )}\end{array}$ & $\begin{array}{l}0.005 \\
(0.468)\end{array}$ & $\begin{array}{l}0.002 \\
(0.811)\end{array}$ & $\begin{array}{l}0.0006 \\
(0.804)\end{array}$ & $\begin{array}{l}0.002 \\
(0.514)\end{array}$ & $\begin{array}{l}0.009 \\
(0.178)\end{array}$ & $\begin{array}{l}0.008 \\
(0.167)\end{array}$ \\
\hline Gov. Expenditure & --- & $\begin{array}{l}0.005 \\
(0.333)\end{array}$ & --- & $\begin{array}{l}0.003 \\
(0.633)\end{array}$ & --- & $\begin{array}{l}0.0008 \\
(0.861)\end{array}$ & --- & $\begin{array}{l}0.008 \\
(0.238)\end{array}$ \\
\hline GDP growth & --- & $\begin{array}{l}0.012 \\
(0.198)\end{array}$ & --- & $\begin{array}{l}0.020 \\
(0.216)\end{array}$ & --- & $\begin{array}{l}0.004 \\
(0.777)\end{array}$ & --- & $\begin{array}{l}0.021 \\
(0.330)\end{array}$ \\
\hline Trade & --- & $\begin{array}{l}0.0002 \\
(0.845)\end{array}$ & --- & $\begin{array}{l}0.0007 \\
(0.573)\end{array}$ & --- & $\begin{array}{l}-0.0004 \\
(0.775)\end{array}$ & --- & $\begin{array}{l}0.0009 \\
(0.529)\end{array}$ \\
\hline Inflation & --- & $\begin{array}{l}0.001 \\
(0.119)\end{array}$ & --- & $\begin{array}{l}-0.001 \\
(0.457)\end{array}$ & --- & $\begin{array}{l}0.001 \\
(0.267)\end{array}$ & --- & $\begin{array}{l}0.0003 \\
(0.689)\end{array}$ \\
\hline $\begin{array}{l}\text { Time effects } \\
\operatorname{AR}(2)\end{array}$ & $\begin{array}{l}\text { Yes } \\
(\mathbf{0 . 6 5 5 )}\end{array}$ & $\begin{array}{l}\text { Yes } \\
(\mathbf{0 . 6 7 3 )}\end{array}$ & $\begin{array}{l}\text { Yes } \\
(\mathbf{0 . 5 2 4 )}\end{array}$ & $\begin{array}{l}\text { Yes } \\
(\mathbf{0 . 6 8 7 )}\end{array}$ & $\begin{array}{l}\text { Yes } \\
(\mathbf{0 . 3 8 1 )}\end{array}$ & $\begin{array}{l}\text { Yes } \\
(\mathbf{0 . 2 9 4 )}\end{array}$ & $\begin{array}{l}\text { Yes } \\
(\mathbf{0 . 7 6 5 )}\end{array}$ & $\begin{array}{l}\text { Yes } \\
(\mathbf{0 . 3 0 7 )}\end{array}$ \\
\hline Sargan OIR & $(0.596)$ & $(\mathbf{0 . 3 0 8})$ & $(0.024)$ & $(\mathbf{0 . 2 2 8})$ & $(0.120)$ & $(0.350)$ & $(0.053)$ & $(0.290)$ \\
\hline Wald (joint) & $\begin{array}{l}\text { 82.210*** } \\
(\mathbf{0 . 0 0 0 )}\end{array}$ & $\begin{array}{l}1065.2 * * * \\
(0.000)\end{array}$ & $\begin{array}{l}\text { 84.379**** } \\
(\mathbf{0 . 0 0 0 )}\end{array}$ & $\begin{array}{l}\text { 1026.0**** } \\
(\mathbf{0 . 0 0 0 )}\end{array}$ & $\begin{array}{l}\text { 49.500**** } \\
(0.000)\end{array}$ & $\begin{array}{l}\text { 1487.1**** } \\
(0.000)\end{array}$ & $\begin{array}{l}133.72 * * * \\
(\mathbf{0 . 0 0 0 )}\end{array}$ & $\begin{array}{l}\text { 3105**** } \\
(\mathbf{0 . 0 0 0 )}\end{array}$ \\
\hline Instruments & 14 & 18 & 14 & 18 & 14 & 18 & 14 & 18 \\
\hline Countries & 51 & 34 & 50 & 34 & 51 & 34 & 50 & 34 \\
\hline Observations & 199 & 118 & 195 & 118 & 199 & 118 & 195 & 118 \\
\hline
\end{tabular}

$* * *, * *$, and * indicate significance at 1\%,5\% and 10\% levels respectively. AR(2): Second Order Autocorrelation test. OIR: Overidentifying Restrictions test. The significance of bold values is twofold. 1) The significance of estimated coefficients and the Wald statistics. 2) The failure to reject the null hypotheses of: a) no autocorrelation in the AR(2) tests and; b) the validity of the instruments in the Sargan OIR test. P-values in bracket. Gov: Government. NODA: Total Net Official Development Assistance. NODAS.D2 (Total): SD2: Distortions by Standard Deviation of the Residuals after first-order autoregressive processes.

Table 6: DAC and MD foreign aid distortions with standard errors

\begin{tabular}{|c|c|c|c|c|c|c|c|c|}
\hline \multirow[b]{3}{*}{ Gov (-1) } & \multicolumn{8}{|c|}{$\begin{array}{c}\text { Dependent variable: Governance } \\
\text { Panel A: Foreign Aid from the Development Assistance Committee (DAC) Countries }\end{array}$} \\
\hline & \multicolumn{2}{|c|}{$\begin{array}{l}\text { Political Governance } \\
\text { (PolGov) }\end{array}$} & \multicolumn{2}{|c|}{$\begin{array}{c}\text { Economic Governance } \\
\text { (EcoGov) }\end{array}$} & \multicolumn{2}{|c|}{$\begin{array}{c}\text { Institutional } \\
\text { Governance (InstGov) }\end{array}$} & \multicolumn{2}{|c|}{$\begin{array}{c}\text { General Governance } \\
\text { (G.Gov) }\end{array}$} \\
\hline & $\begin{array}{l}0.985 * * * \\
(0.000)\end{array}$ & $\begin{array}{l}1.131 * * * \\
(0.000)\end{array}$ & $\begin{array}{l}\text { 1.078**** } \\
(\mathbf{0 . 0 0 0 )}\end{array}$ & $\begin{array}{l}\mathbf{0 . 8 1 0} * * * \\
(\mathbf{0 . 0 0 0 )}\end{array}$ & $\begin{array}{l}\mathbf{0 . 8 3 8} * * * \\
(0.000)\end{array}$ & $\begin{array}{l}0.917 * * * \\
(0.000)\end{array}$ & $\begin{array}{l}1.032 * * * \\
(0.000)\end{array}$ & $\begin{array}{l}\mathbf{0 . 9 5 0 * * *} \\
(\mathbf{0 . 0 0 0 )}\end{array}$ \\
\hline Constant & $\begin{array}{l}-0.050 \\
(0.499)\end{array}$ & $\begin{array}{l}-0.149 \\
(0.170)\end{array}$ & $\begin{array}{l}-0.031 \\
(0.771)\end{array}$ & $\begin{array}{l}0.021 \\
(0.890)\end{array}$ & $\begin{array}{l}0.056 \\
(0.513)\end{array}$ & $\begin{array}{l}0.081 \\
(0.573)\end{array}$ & $\begin{array}{l}0.035 \\
(0.750)\end{array}$ & $\begin{array}{l}-0.075 \\
(0.677)\end{array}$ \\
\hline NODADAC SD2 & $\begin{array}{l}0.002 \\
(0.412)\end{array}$ & $\begin{array}{l}0.013 * * \\
(0.014)\end{array}$ & $\begin{array}{l}0.009 \\
(0.389)\end{array}$ & $\begin{array}{l}0.002 \\
(0.825)\end{array}$ & $\begin{array}{l}0.0002 \\
(0.935)\end{array}$ & $\begin{array}{l}0.002 \\
(0.564)\end{array}$ & $\begin{array}{l}0.011 \\
(0.223)\end{array}$ & $\begin{array}{l}0.010 * \\
(0.097)\end{array}$ \\
\hline Gov. Expenditure & --- & $\begin{array}{l}0.005 \\
(0.306)\end{array}$ & --- & $\begin{array}{l}0.003 \\
(0.683)\end{array}$ & --- & $\begin{array}{l}0.0007 \\
(0.873)\end{array}$ & --- & $\begin{array}{l}0.008 \\
(0.220)\end{array}$ \\
\hline GDP growth & --- & $\begin{array}{l}0.012 \\
(0.194)\end{array}$ & --- & $\begin{array}{l}0.020 \\
(0.229)\end{array}$ & --- & $\begin{array}{l}0.004 \\
(0.742)\end{array}$ & --- & $\begin{array}{l}0.021 \\
(0.307)\end{array}$ \\
\hline Trade & --- & $\begin{array}{l}0.0002 \\
(0.796)\end{array}$ & --- & $\begin{array}{l}0.0007 \\
(0.555)\end{array}$ & --- & $\begin{array}{l}-0.0004 \\
(0.746)\end{array}$ & --- & $\begin{array}{l}0.0007 \\
(0.604)\end{array}$ \\
\hline Inflation & --- & $\begin{array}{l}\mathbf{0 . 0 0 1 *} \\
(0.099)\end{array}$ & --- & $\begin{array}{l}-0.001 \\
(0.464)\end{array}$ & --- & $\begin{array}{l}0.001 \\
(0.273)\end{array}$ & --- & $\begin{array}{l}0.0006 \\
(0.504)\end{array}$ \\
\hline $\begin{array}{l}\text { Time effects } \\
\operatorname{AR}(2)\end{array}$ & $\begin{array}{r}\text { Yes } \\
(\mathbf{0 . 6 8 1 )}\end{array}$ & $\begin{array}{r}\text { Yes } \\
(\mathbf{0 . 6 8 4 )}\end{array}$ & $\begin{array}{r}\text { Yes } \\
(\mathbf{0 . 4 6 6 )}\end{array}$ & $\begin{array}{r}\text { Yes } \\
(\mathbf{0 . 6 5 9 )}\end{array}$ & $\begin{array}{r}\text { Yes } \\
(\mathbf{0 . 3 8 2 )}\end{array}$ & $\begin{array}{r}\text { Yes } \\
(\mathbf{0 . 2 9 3 )}\end{array}$ & $\begin{array}{r}\text { Yes } \\
(\mathbf{0 . 7 7 8 )}\end{array}$ & $\begin{array}{l}\text { Yes } \\
(\mathbf{0 . 3 2 0 )}\end{array}$ \\
\hline Sargan OIR & $(0.527)$ & $(0.288)$ & $(0.027)$ & $(0.205)$ & $(0.125)$ & $(0.360)$ & $(0.060)$ & $(0.269)$ \\
\hline Wald (joint) & $\begin{array}{l}\mathbf{5 2 . 1 8}^{* * * *} \\
(\mathbf{0 . 0 0 0 )}\end{array}$ & $\begin{array}{l}1318.2 * * * \\
(0.000)\end{array}$ & $\begin{array}{l}95.147 * * * \\
(0.000)\end{array}$ & $\begin{array}{l}1019.6^{* * * *} \\
(0.000)\end{array}$ & $\begin{array}{l}\text { 37.637**** } \\
(\mathbf{0 . 0 0 0 )}\end{array}$ & $\begin{array}{l}1818.7 * * * \\
(0.000)\end{array}$ & $\begin{array}{l}130.29 * * * * \\
(0.000)\end{array}$ & $\begin{array}{l}\text { 3194.3*** } \\
(0.000)\end{array}$ \\
\hline Instruments & 14 & 18 & 14 & 18 & 14 & 18 & 14 & 18 \\
\hline Countries & 51 & 34 & 50 & 34 & 51 & 34 & 50 & 34 \\
\hline Observations & 199 & 118 & 195 & 118 & 199 & 118 & 195 & 118 \\
\hline
\end{tabular}




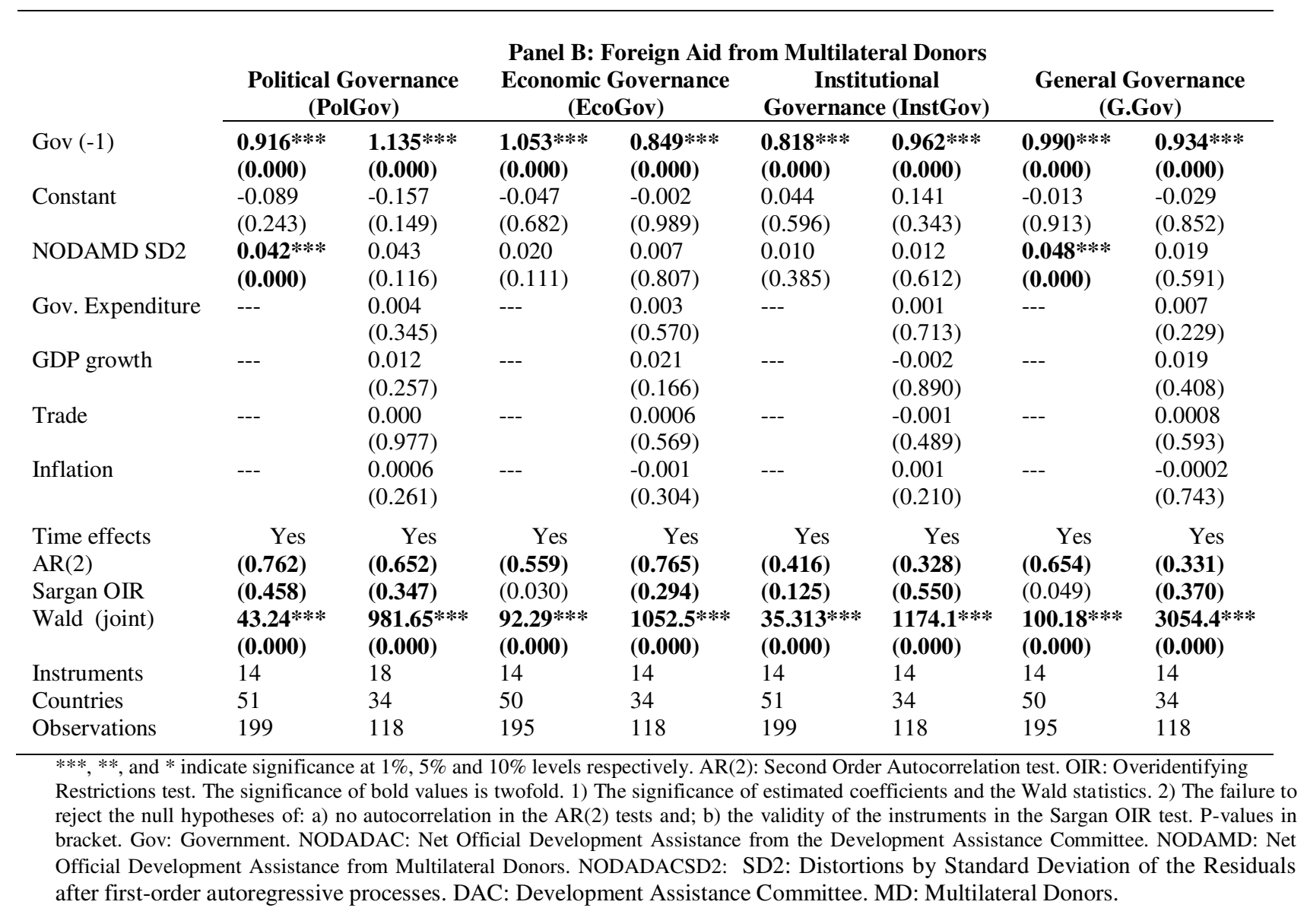

\section{Concluding remarks}

The Kangoye (2013, TDE) findings on the negative nexus between foreign aid unpredictability and governance could seriously affect debates in academic and policy making circles. Using the theoretical underpinnings of the celebrated Eubank (2012, JDS) literature, we have first confirmed Kangoye's findings. Then we have extended the concept of governance from corruption to political, economic, institutional and general versions of the phenomenon. Findings from the extension run counter to those of Kangoye. It follows that in the presence of foreign aid uncertainty, governments could be constrained to improve governance standards in exchange for or anticipation of more dependence on local revenues. This confirms the Eubank hypothesis that aid may dilute the positive appeals of local tax revenues on governance. The empirical evidence 
is based on 53 African countries for the period 1996-2010. Two direct policy implications result.

First, the Kangoye findings for developing countries are relevant for Africa. Second, when the concept of governance is not restricted to corruption, the findings become irrelevant for the continent.

\section{Appendices}

\section{Appendix 1: Definitions of variables}

\begin{tabular}{|c|c|c|}
\hline Variable(s) & Definition(s) & Source(s) \\
\hline Aid1: NODASD1 (Total) & Distortions of Total NODA by Simple Standard Deviation & Author \\
\hline Aid 2: NODADACSD1 & Distortions of NODADAC by Simple Standard Deviation. & Author \\
\hline Aid 3: NODAMDSD1 & Distortions of NODAMD by Simple Standard Deviation & Author \\
\hline Aid1: NODASD2 (Total) & $\begin{array}{l}\text { Distortions of Total NODA by Standard Deviation of the } \\
\text { Residuals after first-order autoregressive process. }\end{array}$ & Author \\
\hline Aid 2: NODADACSD2 & $\begin{array}{l}\text { Distortions of NODADAC by Standard Deviation of the } \\
\text { Residuals after first-order autoregressive process. }\end{array}$ & Author \\
\hline Aid 3: NODAMDSD2 & $\begin{array}{l}\text { Distortions of NODAMD by Standard Deviation of the } \\
\text { Residuals after first-order autoregressive process. }\end{array}$ & Author \\
\hline Political Stability & $\begin{array}{l}\text { Political stability/no violence (estimate): measured as the } \\
\text { perceptions of the likelihood that the government will be } \\
\text { destabilized or overthrown by unconstitutional and } \\
\text { violent means, including domestic violence and } \\
\text { terrorism. }\end{array}$ & World Bank (WDI) \\
\hline Voice \& Accountability & $\begin{array}{l}\text { Voice and accountability (estimate): measures the extent } \\
\text { to which a country's citizens are able to participate in } \\
\text { selecting their government and to enjoy freedom of } \\
\text { expression, freedom of association and a free media. }\end{array}$ & World Bank (WDI) \\
\hline Political Governance & $\begin{array}{l}\text { First Principal Component of Political Stability and } \\
\text { Voice \& Accountability. The process by which those in } \\
\text { authority are selected and replaced. }\end{array}$ & PCA \\
\hline Government Effectiveness & $\begin{array}{l}\text { Government effectiveness (estimate): measures the } \\
\text { quality of public services, the quality and degree of } \\
\text { independence from political pressures of the civil } \\
\text { service, the quality of policy formulation and } \\
\text { implementation, and the credibility of governments' } \\
\text { commitments to such policies. }\end{array}$ & World Bank (WDI) \\
\hline
\end{tabular}


Regulation Quality

Economic Governance

Rule of Law

Corruption Control

Institutional Governance

General Governance

GDP growth

Trade Openness

Government Expenditure

Inflation
Regulation quality (estimate): measured as the ability of the government to formulate and implement sound policies and regulations that permit and promote private sector development.

First Principal Component of Government Effectiveness and Regulation Quality. The capacity of government to formulate \& implement policies, and to deliver services.

Rule of law (estimate): captures perceptions of the extent to which agents have confidence in and abide by the rules of society and in particular the quality of contract enforcement, property rights, the police, the courts, as well as the likelihood of crime and violence.

Control of corruption (estimate): captures perceptions of the extent to which publicpower is exercised for private gain, including both petty and grand forms of corruption, as well as 'capture' of the state by elites and private interests.

First Principal Component of Rule of Law and Corruption-Control. The respect for citizens and the state of institutions

that govern the interactions among them

First principal component of Political Stability, Voice \& Accountability, Government Effectiveness, Regulation Quality, Rule of Law and Corruption-Control.

Gross Domestic Product growth rate (annual \%)

Exports plus Imports of Commodities (\% of GDP)

Government Final Consumption Expenditure(\% of GDP)

Consumer Price Index (annual \%)
World Bank (WDI)

PCA

World Bank (WDI)

World Bank (WDI)

PCA

PCA

World Bank (WDI)
World Bank (WDI)
World Bank (WDI)
World Bank (WDI)

WDI: World Bank Development Indicators. GDP: Gross Domestic Product. PCA: Principal Component Analysis. NODA: Net Official Development Assistance. NODADAC: NODA from the Development Assistance Committee (DAC) countries. NODAMD: NODA from Multilateral Donors. SD1: Distortions by Simple Standard Deviation. SD2: Distortions by Standard Deviation of the Residuals after first-order autoregressive processes.

\section{Appendix 2: Summary statistics}

First Distortions from Total NODA

First Distortions from Total NODADAC

First Distortions from Total NODADMD

Second Distortions from Total NODA

Second Distortions from Total NODADAC

Second Distortions from Total NODADMD

Political Governance (PolGov)

Economic Governance (EcoGov)

Institutional Governance (InstGov)

General Governance (G.Gov)

Corruption (Corruption Perception Index)

GDP growth

Trade Openness

Government Expenditure

Inflation

\begin{tabular}{ccccc} 
Mean & S.D & Min & Max & Obs. \\
\hline 2.841 & 6.460 & 0.001 & 64.113 & 250 \\
1.868 & 4.790 & 0.0005 & 44.404 & 250 \\
1.397 & 2.712 & 0.0006 & 29.353 & 250 \\
3.409 & 8.106 & 0.005 & 91.927 & 250 \\
2.201 & 6.333 & 0.001 & 68.826 & 250 \\
1.678 & 2.714 & 0.000 & 29.906 & 250 \\
-0.016 & 1.291 & -3.204 & 2.621 & 264 \\
0.049 & 1.310 & -3.019 & 3.290 & 254 \\
0.008 & 1.378 & -3.879 & 3.179 & 264 \\
0.108 & 2.095 & -5.139 & 5.086 & 254 \\
3.005 & 1.064 & 1.066 & 6.100 & 181 \\
4.755 & 5.587 & -11.272 & 49.367 & 254 \\
78.340 & 39.979 & 20.980 & 250.95 & 247 \\
4.495 & 8.064 & -17.387 & 49.275 & 164 \\
56.191 & 575.70 & -45.335 & 8603.3 & 230
\end{tabular}

S.D: Standard Deviation. Min: Minimum. Max: Maximum. Obs: Observations. NODA: Net Official Development Assistance. DAC: Development Assistance Committee. SD1: Distortions by Simple Standard Deviation. SD2: Distortions by Standard Deviation of the Residuals after first-order autoregressive processes. 
Appendix 3: Correlation Analysis

\begin{tabular}{|c|c|c|c|c|c|c|c|c|c|c|c|c|c|c|c|}
\hline \multicolumn{4}{|c|}{ Control Variables } & \multicolumn{6}{|c|}{ Foreign Aid Distortions } & \multicolumn{5}{|c|}{ Governance } & \multirow[b]{3}{*}{$\mathrm{GDPa}$} \\
\hline GDPg & Trade & Gov.E & Inflation & SD1Aid1 & SD1Aid2 & SD1Aid3 & SD2Aid1 & SD2Aid2 & SD2Aid3 & PolGov & EcoGov & InstGov & G.Gov & Corruption & \\
\hline \multirow[t]{15}{*}{1.000} & 0.179 & 0.254 & -0.132 & 0.219 & 0.193 & 0.166 & 0.145 & 0.091 & 0.109 & -0.012 & -0.041 & -0.084 & -0.049 & -0.056 & \\
\hline & 1.000 & -0.070 & 0.024 & 0.082 & 0.050 & 0.047 & 0.105 & 0.091 & -0.032 & 0.202 & 0.089 & 0.207 & 0.174 & 0.209 & Trade \\
\hline & & 1.000 & -0.243 & 0.014 & 0.024 & 0.072 & 0.028 & 0.028 & 0.051 & -0.040 & 0.007 & 0.023 & -0.003 & -0.095 & Gov. E \\
\hline & & & 1.000 & -0.004 & 0.011 & -0.016 & -0.003 & 0.006 & 0.016 & -0.114 & -0.169 & -0.136 & -0.149 & -0.054 & Inflation \\
\hline & & & & 1.000 & 0.921 & 0.793 & 0.949 & 0.878 & 0.678 & -0.157 & -0.293 & -0.215 & -0.244 & -0.130 & SD1Aid1 \\
\hline & & & & & 1.000 & 0.528 & 0.901 & 0.946 & 0.459 & -0.160 & -0.279 & -0.224 & -0.242 & -0.129 & SD1Aid2 \\
\hline & & & & & & 1.000 & 0.718 & 0.515 & 0.902 & -0.105 & -0.252 & -0.157 & -0.191 & -0.132 & SD1Aid3 \\
\hline & & & & & & & 1.000 & 0.945 & 0.650 & -0.109 & -0.251 & -0.179 & -0.198 & -0.118 & SD2Aid1 \\
\hline & & & & & & & & 1.000 & 0.452 & -0.115 & -0.228 & -0.182 & -0.191 & -0.112 & SD2Aid2 \\
\hline & & & & & & & & & 1.000 & -0.074 & -0.234 & -0.153 & -0.175 & -0.161 & SD2Aid3 \\
\hline & & & & & & & & & & 1.000 & 0.758 & 0.819 & 0.901 & 0.745 & PolGov \\
\hline & & & & & & & & & & & 1.000 & 0.878 & 0.945 & 0.822 & EcoGov \\
\hline & & & & & & & & & & & & 1.000 & 0.957 & 0.895 & InstGov \\
\hline & & & & & & & & & & & & & 1.000 & 0.875 & G.Gov \\
\hline & & & & & & & & & & & & & & 1.000 & Corruption \\
\hline
\end{tabular}

GDPg: GDP growth rate. Gov. E: Government Expenditure. Aid1: Total Net Official Development Assistance (NODA). Aid2: NODA from the DAC countries. Aid3: NODA from Multilateral Donors. SD1: Distortions by Simple Standard Deviation. SD2: Distortions by Standard Deviation of the Residuals after first-order autoregressive processes. PolGov: Politcal Governance. EcoGov: Economic Governance. InstGov: Institutional Governance. G.Gov: General Governance. 


\section{References}

Andrés, A. R., \& Asongu, S. A., (2013). "Fighting software piracy: which governance tools matter in Africa?", Journal of Business Ethics, 118(3), pp. 667-682.

Andrés, A. R., Asongu, S. A., \& Amavilah, V. H. S., (2014). "The Impact of Formal Institutions on Knowledge Economy", Journal of the Knowledge Economy: Forthcoming. http://link.springer.com/article/10.1007\%2Fs13132-013-0174-3

Arellano, M., \& Bond, S. (1991) "Some tests of specification for panel data: Monte Carlo evidence and an application to employment equations" The Review of Economic Studies 58, 277297.

Arellano, M., \& Bover, O. (1995) "Another look at the instrumental variable estimation of errorcomponents models" Journal of Econometrics 68, 29-52.

Asongu, S. A., (2012). "Government quality determinants of stock market performance in African countries”, Journal of African Business, 13(3), pp. 183-199.

Asongu, S. A., (2014a). "On taxation, political accountability and foreign aid: empirics to a celebrated literature", African Governance and Development Institute Working Paper.

Asongu, S. A., (2014b). "Globalization, (fighting) corruption and development: how are these phenomena linearly and nonlinearly related in wealth-effects?", Journal of Economic Studies, 41(3), pp. 346-369.

Asongu, S. A., \& Jellal, M., (2013). "On the channels of foreign aid to corruption", Economics Bulletin, 33(3), pp. 2191-2201.

Blundell, R., \& Bond, S., (1998). "Initial conditions and moment restrictions in dynamic panel data models", Journal of Econometrics, 87(1), pp.115-143.

Bond, S., Hoeffler, A., \& Tample, J., (2001). "GMM Estimation of Empirical Growth Models", University of Oxford.

Collier, P. (2007). The Bottom Billion: Why the Poorest Countries Are Failing and What Can Be Done About It ?, Oxford University Press.

Eubank, N. (2012). "Taxation, Political Accountability and Foreign Aid: Lessons from Somaliland", Journal of Development Studies, 48(4), pp. 465-480.

Kangoye, T., (2013). "Does Aid Unpredictability Weaken Governance? Evidence From Developing Countries", The Developing Economies, 51(2), pp. 121-144.

Kaufmann, D., Kraay, A., \& Mastruzzi, M., (2010). "The worldwide governance indicators: Methodology and analytical Issues”. World Bank Policy Research Working Paper No 5430. 
Khandelwal, P., \& Roitman, A., (2012). "The Economics of Political Transitions: Implications for the Arab Spring", IMF Working Paper No. 13/69.

Lalountas, D.A., Manolas, G.A., \& Vavouras, I.S., (2011), “ Corruption, globalization and development: How are these three phenomena related?", Journal of Policy Modeling, 33, pp.636648.

Moyo, D. (2009). Dead Aid: Why Aid Is Not Working and How There is Another Way for Africa. New York: Farrar, Straus and Giroux.

Stoddard, E. (2014, February). "Somaliland blessed by dodging aid 'curse': minister", Reuters (South Africa), http://mobile.reuters.com/article/idUSBREA1316Y20140204?irpc=932 (Accessed: 23/02/2014). 